Review article

\title{
ANTIBABESIAL TREATMENT PROTOCOLS AGAINST CANINE BABESIOSIS
}

\author{
W.R. Bandula Kumara B.V.Sc., Ph.D. \\ Veterinary Teaching Hospital, Department of Veterinary Clinical Science, \\ Faculty of Veterinary Medicine and Animal Science, University of Peradeniya, Sri Lanka
}

\begin{abstract}
SUMMARY: Canine babesiosis is an important tick-borne disease caused by hemoprotozoan parasites of the genus Babesia, and the predominant species infecting dogs is Babesia gibsoni. The disease has been identified worldwide and is now recognized as a serious emergent disease in dogs. Although the incidence of canine babesiosis has been increasing, effective and crucial therapeutic modality is unavailable. The conventional antibabesial drugs including diminazene aceturate, pentamidine, and imidocarb dipropionate could not eliminate the parasites from infected dogs. Newer therapeutic modality for canine babesiosis using drugs such as clindamycin, atovaquone and multiple drug combinations has been suggested. Although these therapeutic modalities are beneficial and seem to partially provide a permanent cure, the treatments failures often occur. Thus, broad understanding of treatment protocols are required when implementing an appropriate treatment plan against canine babesiosis. The goal of this review is to provide veterinary practitioners with guidelines for successful treatment against canine babesiosis. It is expected that the review will answer the most frequently asked questions posed by veterinary practitioners.
\end{abstract}

\section{BACKGROUND}

Canine babesiosis is an important tickborne disease caused by hemoprotozoan parasites of the genus Babesia, and the predominant species infecting dogs is Babesia gibsoni (Bandula et al.,2012a, 2012b, Yamasaki et al.,2002, 2003, 2007, 2011). The disease has been identified worldwide and is now recognized as a serious emergent disease in dogs (Birkenheuer et al.,1999, Matsuu et al.,2006). B. gibsoni in particular, has been reported to occur endemically in Asia, Africa, Europe, North America and Australia (Collett, 2000, Matsuu et al.,2008). Many drugs have been utilized for the treatment of canine babesiosis, such as diminazene aceturate (Berenil), imidocarb dipropionate (Imizol), phenamidine isethionate, pentamidine isethionate, trypan blue, primaquone and quinuronium sulfate (Bandula et al.,2012a, 2012b, Birkenheuer et al.,2003, Matsuu et al.,2008, Sakuma et al.,2009); however, none of these drugs have been proven to be effective for the elimination of B. gibsoni organisms from infected dogs (Bandula et al.,2012a, 2012b, Bielawski et al.,2000). Some antibabesial drugs can reduce the severity of clinical signs and the mortality associated with the disease. Treatment or spontaneous recovery from an acute infection frequently fails to clear the organism from the host, resulting in a carrier stage (Wulansari et al.,2003).

\section{CONVENTIONAL ANTIBABESIAL DRUGS USED AGAINST CANINE BABESIOSIS}

Diminazene aceturate and aromatic pentamidine analogues, such as DB75 ([2, 5-bis (4amidinophenyl) furan]) and DB820 (6-[5-(4amidinophenyl)-furan-2-yl] nicotinamidine) have been used as therapeutic agents for many parasite diseases for several decades. Currently, the most common conventional antibabesial drug used in $B$. gibsoni infection is diminazene aceturate. A single intramuscular injection of diminazene aceturate at a dose of $3.5 \mathrm{mg} / \mathrm{kg}$ (Breitschwerdt et al.,1990) to 5 $\mathrm{mg} / \mathrm{kg}$ (Birkenheuer et al.,1999) has demonstrated efficacy against B. gibsoni infection (Wulansari et al.,2003). In certain cases, diminazene aceturate needs to be repeated at 5 day intervals until clinical signs subside and parasites disappear from peripheral blood. However, it has been recommended that diminazene at $4.2 \mathrm{mg} / \mathrm{kg}$ intramuscular dose should not be repeated within a 21-days period as plasma concentrations remained even at 21 days (Miller et al 2005). In general, diamidine analogues interact with the minor groove of the DNA double helix containing high adenine (A) + thiamine (T) content (Basselin et al., 1998). This interaction would interfere with the transcription, replication and stabilization of DNA, leading to death of the parasites (Basselin et al.,1998, Bell et al.,1991, Coates et al.,2002, Lane et al.,1991, Mathis et al.,2007, Purfield et $a l ., 2009)$. On the other hand, diminazene aceturate has a low therapeutic index, thus potentially dangerous and shows a propensity to develop 
severe cerebral toxicity with classic cerebellar sulci haemorrhages particularly in Alaskan malamute (Donghyun et al.,2014). Furthermore, Dachshund, Golden Retriever, Labrador, Doberman and Rottweiler are more susceptible to diminazene aceturate toxicity. The clinical signs associated with diminazene toxicity are depression or stupor, continuous vocalization, ataxia, opisthotonos, extensor rigidity, nystagmus and seizures. It often causes fatal nervous complication after $24-48$ hours of overdose (Milner et al.,1997). Although higher dose, repeated doses and intravenous administration of diminazene can induce toxicity more easily, toxicity can occur by lower dose, single dose and intravenous administration in dogs (Donghyun et al.,2014).

Although diminazene aceturate has been used as a first-line agent for the treatment of $B$. gibsoni infection in dogs (Sakuma et al.,2009), it cannot completely eliminate $B$. gibsoni from infected dogs, and relapses often occur (Farwell et al., 1982, Hwang et al.,2010a, Matsuu et al.,2008, Wulansari et al.,2003). It is reported that one of the reasons for the relapse of canine babesiosis might be the development of diminazene aceturate resistance in Babesia parasites (Collett, 2000, Matsuu et al.,2006, Sakuma et al.,2009). Diamidine resistance has been well described in Trypanosoma (Teka et al.,2011) and Leishmania species (Sereno et al.,1997). It is reported that diamidine is a substrate of P2 nucleoside transporters operative in $T$. brucei (Carter et al.,1995, Teka et al.,2011). The loss of that transporter and high-affinity pentamidine transporter functions in African trypanosomes has been partially implicated in resistance to diamidine (Carter et al.,1995, Teka et al.,2011). In addition, numerous dehydrogenases and F1F0-ATPase in mitochondria are decreased in diamidine-resistant parasites (Basselin et al.,2002, Mukherjee et al.,2006, Soto et al.,2009). However, the mechanisms of diamidine resistance in Babesia are less known and thus need to be investigated.

Imidocarb dipropionate (Imizol) is another conventional therapy for canine babesiosis. It is given at $5-6 \mathrm{mg} / \mathrm{kg}$ by subcutaneous or intramuscular injection (Abdullah et al.,1984, Uilenberg et al.,1981). Very often two doses of imidocarb are given 2 weeks apart. The deep muscular injection is much safer because the drug causes severe inflammation (Abdullah et al.,1984). Imidocarb reduces morbidity and mortality of the parasite but ineffective for clearance of $B$. gibsoni. Some dogs treated by this drug recover very rapidly but others do not. Those that do recover may become chronic carriers that transmit the disease to other dogs. There are adverse effects of imidocarb such as difficulty in breathing, tachycardia, weakness and profuse diarrhea, pulmonary congestion, oedema, splenomegaly, hepatomegaly and renomegaly (Abdullah et al., 1984). They suggested that these adverse effects of imidocarb may be due to excessive acetylcholine action (Abdullah et al.,1984). Nevertheless, imidocarb is generally considered as a safe drug for canine babesiosis especially for puppies and geriatric patients. In addition, when dogs are simultaneously infected with a second parasite, Ehrlichia canis, imidocarb can affect both parasites (Pasa et al.,2011).

\section{USE OF MACROLIDE ANTIBIOTICS AND ANTIFUNGAL COMPOUNDS AS ANTIBABESIAL DRUGS}

Yamasaki et al.,(2011b) have reported that nystatin; a membrane-active polyene macrolide antibiotic and an antifungal compound could destroy B. gibsoni by its ionophorous activity. The drug operates as a channel-forming ionophore and is membranolytic due to its lipid binding activity (Wiehart et al.,2006, Yamasaki et al.,2011b). Nystatin is not given intravenously to dogs because of its toxicity, attributed to the ability of polyene macrolides to bind cholesterol in mammalian cell membranes, albeit with lower affinity (Wiehart et al.,2006). Ionophorous antibiotics valinomycin and salinomycin-Na, have exhibited a strong in vitro effect against B. gibsoni (Yamasaki et al.,2005) by modifying the intracellular concentrations of monovalent cations. This action may activate transporters of monovalent cations, such as Na-KATPase, and increase the consumption of adenosine triphosphate, resulting in the depletion of intracellular ATP (Yamasaki et al.,2005). However, valinomycin and salinomycin-Na have no specificity, as they affect both the parasites and the host cells in which intracellular concentrations of monovalent cations are regulated by the function of an active transporter, and therefore, they are ineffective as therapeutics against $B$. gibsoni infection.

Amphotericin B is a membrane-active polyene macrolide antibiotic which has antibabesial effect. The interaction of such compounds with sterols in bilayer cell membranes can lead to membrane damage and ultimately cell lysis (Yamasaki et al.,2014). Although experimentally 0.5 and $1 \mathrm{mg} / \mathrm{kg}$ amphotericin B administered by the intravenous route to $B$. gibsoni-infected dogs has reduced parasitemia, recurrence of parasitemia had been observed, indicating that amphotericin B did not eliminate parasites completely (Yamasaki et al.,2014). Furthermore, blood urea nitrogen and creatinine levels of dogs were markedly elevated after the administration of $1 \mathrm{mg} / \mathrm{kg}$ amphotericin B (Yamasaki et al.,2014). Accordingly, amphotericin $\mathrm{B}$ has in vivo activity against $B$. gibsoni; however, it does not eliminate parasites from infected dogs and affects kidney function at a high dose. Thus, 
amphotericin B needs to be used with care especially in patients with impaired renal functions.

Clotrimazole and ketoconazole are imidazole derivatives and antifungal agents that are also reported to inhibit the in vitro growth of Babesia species, including B. equi, B. caballi (Bork et al.,2003a), B. bigemina, and B. bovis (Bork et $a l ., 2003 b$ ). However, the sensitivity of $B$. gibsoni to clotrimazole and ketoconazole was intermediate compared to other Babesia species (Matsuu et al.,2008). It has been suggested that their antiprotozoal activity might be due to their ability to cause major changes in calcium ion fluxes (Matsuu et al.,2008).

\section{ATOVAQUONE AND ITS COMBINATION AS TREATMENTS FOR CANINE BABESIOSIS}

Atovaquone is a novel antiprotozoal compound that has broad-spectrum activity against protozoan pathogens including Babesia species (Birkenheuer et al.,2004, Matsuu et al.,2006, 2008, Sakuma et al.,2009). Atovaquone is commercially available in 2 formulations: a single drug formulation and a 2.5: 1 combination with proguanil hydrochloride (Birkenheuer et al.,2004). Atovaquone blocks mitochondrial electron transfer and thereby the production of energy by the parasites (Matsuu et al.,2006). Proguanil is metabolized into its active metabolite, cycloguanil, which blocks dihydrofolate reductase and enzymes required for making pyrimidine which is needed for DNA synthesis (Birkenheuer et al.,2004). However, the therapeutic protocol has several problems in canine babesiosis, such as a relatively longer time to show clinical effectiveness and adverse effects such as vomiting and diarrhea. Moreover, atovaquone is expensive and not commonly available in many countries including Sri Lanka. Recurrence of disease and decreased sensitivity of Babesia to therapy have been reported when atovaquone alone was used (Matsuu et al.,2006, Birkenheuer et al.,2004). Mutation of the cytochrome $b$ gene, which is located in the mitochondrial genome, has been described as a cause for less susceptibility of $B$. gibsoni to atovaquone (Matsuu et al.,2006). Meanwhile, it has been demonstrated that an atovaquoneazithromycin drug combination is an effective treatment for dogs that are chronically infected with B. gibsoni (Matsuu et al.,2006, 2008, Sakuma et al.,2009). Azithromycin is a macrolide antibiotic, which has also been confirmed to exhibit activity against Plasmodium species in vitro and in vivo (Nakornchai et al.,2006). The lack of adverse clinical, hematological and biochemical abnormalities reported with the combination treatment is promising, but many studies are underway to fully assess the safety of the atovaquone-azithromycin drug combination in dogs.

\section{MULTIPLE DRUG COMBINATIONS FOR CANINE BABESIOSIS}

An alternative therapy of additive and synergistic strategy is combination therapies with clindamycin-metronidazole-doxycycline at dose rates of clindamycin at $25 \mathrm{mg} / \mathrm{kg}$, metronidazole at $15 \mathrm{mg} / \mathrm{kg}$ and doxycycline at $5 \mathrm{mg} / \mathrm{kg}$ orally with frequency of 12 hours for 10 days (Nandini et al.,2016). This combination boosts the innate immunity and is known as the Marshall Protocol. That is probably the reason for lack of adverse reactions and the dogs show clinical and haematological improvement from few days of treatment onwards and recover uneventfully by the end of therapeutic protocol (Nandini et al.,2016, Wulansari et al.,2003) Clindamycin is a semisynthetic derivative of lyncomycin. The in vitro activities of clindamycin against $B$. divergens were demonstrated with an IC50 value of 2200 $3400 \mathrm{mg} / \mathrm{l}$, which corresponds to $4.36-6.73 \mathrm{mM}$ (Brasseur et al.,1998). However, Matsuu et al.,(2008) have observed that the activity of clindamycin against $B$. gibsoni was $16-24$ times lower than that against $B$. divergens. Thus, it may explain that treatment with clindamycin alone is not sufficient to eliminate the clinical symptoms and Babesia organisms from the peripheral blood of B. gibsoni infected dogs (Wulansari et al.,2003). Nevertheless, it is also been suggested that clindamycin stimulate humoral and cellular immunity against Babesia infection and results in improvement in clinical condition (Hwang et al.,2010, Nandini et al.,2016, Wulansari et al.,2003). For a long time, tetracycline antibiotics; doxycycline hydrochloride and minocycline hydrochloride have been known to exhibit activity against Babesia parasites such as $B$. divergens (Losson et al.,1989) and B. canis has been recognized only in vivo (Vercammen et al.,1996). Tetracycline, macrolide and lyncomycin exhibit inhibitory effects against the apicomplex protozoa by probably targeting the apicoplast (Dahl et al.,2008). Metronidazole is a nitroimidazole compound used commonly as an antitrichomonal. Although metronidazole was reported to have been used as part of the combination therapy (Suzuki et al.,2007), no activity was observed in in vitro studies of B. gibsoni (Hwang et al.,2010a, Matsuu et al.,2008). Nevertheless, the combined therapy of clindamycin, metronidazole and doxycycline is an efficacious alternative treatment strategy for chronic clinical babesiosis with less adverse effects that has been observed in the limited research conducted so far. Meanwhile, there are many reports of development of antibiotic resistance for those antibiotics, for example, 
emergence of clindamycin-resistant in methicillinresistant Staphylococcus aureus (Rich et al.,2005), metronidazole-resistant Trichomonas vaginalis (Cudmore et al.,2004) and doxycycline-resistant strains of Streptococcus (Chalker, et al.,2012) in animals. Thus, combination treatments of these antibiotics against babesiosis warrant further studies due to possible emergence of not only antibiotic-resistant bacteria but also Babesia.

Another drug combination that is used to treat naturally occurring canine babesiosis is a doxycycline-enrofloxacin-metronidazole combination with and without diminazene aceturate (Lin et al.,2010, Vial et al.,2006). The overall efficacy of combination of doxycyclineenrofloxacin-metronidazole in conjunction with and without administration of diminazene aceturate for B. gibsoni is $85.7 \%$ and $83.3 \%$, respectively, with a mean recovery time of 24.2 and 23.5 days, respectively (Lin et al.,2010). This means concomitant use of intramuscular diminazene aceturate may not improve the efficacy of a doxycycline-enrofloxacin-metronidazole combination in management of canine babesiosis caused by $B$. gibsoni.

\section{CONCLUSIONS}

Although the incidence of canine babesiosis has been increasing, effective and crucial therapeutic modality is unavailable. Single drug treatment or combined treatment with diminazene aceturate, atovaquone, clindamycin, metronidazole, doxycycline, and pentamidine do not eliminate the parasites from the peripheral blood. Thus, a broad understanding of treatment protocols is required when implementing an appropriate treatment plan against canine babesiosis. Furthermore, it is also important to consider factors such as drug availability, dosage regimes and their adverse effects, convenience to use, and the cost. Importance of follow up treatments needs to be explained to the clients as a part of the management strategies of canine babesiosis. The combination therapy might be more effective rather than a single therapy. However, the combination treatments are generally used as the second-line of treatments when conventional therapies fail. Many studies are required for analyzing the correlations of these drugs when used in combination with each other, and to determine the best efficient composition for the inhibition of the growth of parasite. Most importantly, possible relapses and the development of drug-resistant isolates are matters of concern. Thus, the development of novel, more effective antibabesial drugs against canine babesiosis is urgently required.

\section{ACKNOWLEDGEMENTS}

The author is grateful to Professor Indira D. Silva, Dr D. R. Anuruddhika Dissanayake and Dr K. A. Nayana Wijayawardhane who have encouraged writing this review.

\section{REFERENCES}

Abdullah, A. S., Sheikh-Omar, A. R., Baggot, J. D. and Zamri, M. (1984). Adverse effects of imidocarb dipropionate (Imizol) in a dog. Veterinary Research Communication, 8(1): 5559.

Bandula, W. R. K., Yamasaki, M., Hwang, S. J., Nakamura, K., Sasaki, N., Murakami, M., Tamura, Y., Lim S. Y., Ohta, H. and Takiguchi, M. (2012a). Involvement of mitochondrial genes of Babesia gibsoni in resistance against diminazene aceturate. Journal of Veterinary Medical Science, 74(9): 1139-1148.

Bandula, W. R. K., Yamasaki, M., Hwang, S. J., Nakamura, K., Sasaki, N., Murakami, M., Tamura, Y., Lim S. Y., Ohta, H. and Takiguchi, M. (2012b). Analysis of energy generation and glycolysis pathway in diminazene aceturate-resistant Babesia gibsoni isolate in vitro. Japanese Journal of Veterinary Research, 60: 51-61.

Basselin, M., Badet-Denisot, M. and Robert-Gero, M. (1998). Modification of kinetoplast DNA minicircle composition in pentamidineresistant Leishmania. Acta Tropica, 70: 43-61.

Basselin, M., Denise, H., Coombs, G. H. and Barrett, M. P. (2002). Resistance to pentamidine in Leishmania exicana involves exclusion of the drug from the mitochondrion. Antimicrobial Agents and Chemotherapy, 46: 3731-3738.

Bell, C. A., Cory, M., Fairley, T. A., Hall, J. E. and Tidwell, R. R. (1991). Structure-activity relationships of pentamidine analogs against Giardia lamblia and correlation of antigiardial activity with DNA-binding affinity. Antimicrobial Agents and Chemotherapy, 35: 1099-1107.

Bielawski, K., Galicka, A., Bielawska, A. and Sredzinska, K. (2000). Inhibitory effects of pentamidine analogues on protein biosynthesis in vitro. Acta Biochimica Polonica, 47: 113120.

Birkenheuer, A. J., Levy, M. G., Savary, K. C., Gager, R. B. and Breitschwerdt, E. B. (1999). Babesia gibsoni infections in dogs from North Carolina. Journal of the American Hospital Association, 35: 125-128.

Birkenheuer, A. J., Levy, M. G. and Breitschwerdt, E. B. (2003). Development and evaluation of a seminested PCR for detection and differentiation of Babesia gibsoni (Asian 
genotype) and B. canis DNA in canine blood samples. Journal Clinical Microbiology, 41: 4172-4177.

Birkenheuer, A. J., Levy, M. G. and Breitschwerdt, E. B. (2004). Efficacy of combined atovaquone and azithromycin for therapy of chronic Babesia gibsoni (Asian Genotype) infections in dogs. Journal Veterinary Internal Medicine, 18: 494-498.

Brasseur, P., Lecoublet, S., Kapel, N., Favennec, L. and Ballet, J. (1998). In vitro evaluation of drug susceptibilities of Babesia divergens isolates. Antimicrobial Agent and Chemotherapy, 42: 818-820.

Breitschwerdt, E. B. (1990). Babesiosis. In: infectious diseases of the dog and cat, First edition., Greene, C. E. ed., W. B. Saunders Co. Press, Philadelphia, 796-803.

Bork, S., Yokoyama, N., Maysuo, T., Claveria, F.G., Fujisaki, K. and Igarashi, I. (2003a). Clotrimazole, ketoconazole, and clodinafoppropargyl as potent growth inhibitors of equine Babesia parasites during in vitro culture. Journal of Parasitology, 89: 604-606.

Bork, S., Yokoyama, N., Matsuo, T., Claveria, F.G., Fujisaki, K. and Igarashi, I. (2003b). Clotrimazole, ketoconazole, and clodinafoppropargyl inhibit the in vitro growth of Babesia bigemina and Babesia bovis (Phylum Apicomplexa). Parasitology, 127: 311-315.

Carter, N. S., Berger, B. J. and Fairlamb, A. H. (1995). Uptake of diamidine drugs by the P2 nucleoside transporter in melarsen-sensitive and -resistant Trypanosoma brucei brucei. Journal of Biological Chemistry, 270: 2815328157.

Chalker, V. J., Waller, A., Webb, K., Spearing, E., Crosse, P., Brownlie, J. and Erles, K. (2012). Genetic diversity of Streptococcus equi subspecies zooepidemicus and doxycycline resistance in kenneled dogs. Journal of Clinical Microbiology,50 (6): 2134-2136.

Coates, L., Ikpeazu, E. V., Chen, Y. and Valenzuela, M. S. (2002). Inhibition of DNA replication by berenil in plasmids containing poly(dA)poly(dT) sequences. Plasmid, 47: 120-128.

Collett, M. G. (2000). Survey of canine babesiosis in South Africa. Journal of South African Veterinary Association, 71: 180-186.

Cudmore, S. L., Delgaty, K. L., HaywardMcClelland, S. F., Petrin, D. P. and Garber G. E. (2004). Treatment of infections caused by metronidazole-resistant Trichomonas vaginalis. Clinical

Microbiology Review, 17(4): 783-793.

Dahl, E.L. and Rosenthal, P.L. (2008). Apicoplast translation, transcription and genome replication: targets for antimalarial antibiotics. Trends Parasitology, 24: 279-284.

Donghyun, H., Won-Kyoung, Y. and Changbaig, H. (2014). Cerebellar encephalopathy from diminazene aceturate (beneril) toxicity in a dog. Korean Journal of Veterinary Research, 54(3): 193-196.

Farwell, G. E., LeGrand, E. K. and Cobb, C. C. (1982). Clinical observations on Babesia gibsoni and Babesia canis infections in dogs. Journal of the American Hospital Association, 180: 507-511.

Hwang, S. J., Yamasaki, M., Nakamura, K., Sasaki, N., Murakami, M., Bandula, W. R. K., Ohta, H., Maede, Y. and Takiguchi, M. (2010a). Development and characterization of a strain of Babesia gibsoni resistant to diminazene aceturate in vitro. Journal of Veterinary Medical Science, 72: 765-771.

Hwang, S. J., Yamasaki, M., Nakamura, K., Sasaki, N., Murakami, M., Bandula, W. R. K., Ohta, H., Maede, Y. and Takiguchi, M. (2010b). Reduced transcript levels of the heat shock protein 70 gene in diminazene aceturateresistant Babesia gibsoni variants under low concentrations of diminazene aceturate. Japanese Journal Veterinary Research, 58: 155-164.

Irwin, P. J. (2010). Canine Babesiosis. Veterinary Clinics of North America: Small Animal Practice, 40(6): 1141-1156.

Lane, A. N., Jenkins, T. C., Brown, T. and Neidle, S. (1991). Interaction of berenil with the EcoRI dodecamer d(CGCGAATTCGCG) 2 in solution studied by NMR. Biochemistry, 30: 1372-1385.

Lin, M. Y. and Huang, H. P. (2010). Use of a doxycycline-enrofloxacin-metronidazole combinationwith/without diminazene diaceturate to treat naturally occurring canine babesiosis caused by Babesia gibsoni. Acta Veterinaria Scandinavica, 52: 27-30.

Losson, B. and Patz, R. (1989). Babesia divergens: activity of long-acting oxytetracycline in the gerbil, Meriones unguiculatus. Annals of veterinary research, 20: 501-507.

Mathis, A. M., Bridges, A. S., Ismail, M. A., Kumar, A., Francesconi, I., Anbazhagan, M., $\mathrm{Hu}$, Q., Tanious, F. A., Wenzler, T. and Saulter, J. (2007). Diphenyl furans and aza analogs: effects of structural modification on in vitro activity, DNA binding, and accumulation and distribution in trypanosomes. Antimicrobial Agents Chemotherapy, 51: 2801-2810.

Matsuu, A., Miyamoto, K., Ikadai, H., Okano, S. and Higuchi, S. (2006). Short report: cloning of the Babesia gibsoni cytochrome $b$ gene and isolation of three single nucleotide polymorphisms from parasites present after 
atovaquone treatment. The American Journal of Tropical Medicine and Hygiene, 74: 593597.

Matsuu, A., Yamasaki, M., Xuan, X., Ikadai, H. and Hikasa, Y. (2008). In vitro evaluation of the growth inhibitory activities of 15 drugs against Babesia gibsoni (Aomori strain). Veterinary Parasitology, 157: 1-8.

Miller, D. M., Swan, G. E., Lobetti, R. G. and Jacobson, L. S. (2005). The pharmacokinetics of diminazene aceturate after intramuscular administration in healthy dogs. Journal of South African Veterinary Association, 76(3): 146-150.

Milner, R. J., Reyers, F., Taylor, J. H. and Van den Berg, J. S. (1997). The effect of diminazene aceturate on cholinesterase activity in dogs with canine babesiosis. Journal of South African Veterinary Association, 68(4): 111113.

Mukherjee, A., Padmanabhan, P. K., Sahani, M. H., Barrett, M. P. and Madhubala, R. (2006). Roles for mitochondria in pentamidine susceptibility and resistance in Leishmania donovani. Molecular and Biochemical Parasitology, 145: 1-10.

Nandini, M. K., Vishwakarma, P. and Kamran, C. A. (2016). New Therapeutic Protocol for Canine Babesiosis: A Case Report. Journal of Dairy, Veterinary and Animal Research, 3: 13.

Nakornchai, S. and Konthiang, P. (2006). Activity of azithromycin or erythromycin in combination with antimalarial drugs against multidrug-resistant Plasmodium falciparum in vitro. Acta Tropica, 100: 185-191.

Pasa, S., Voyvoda, H., Karagenc, T., Atasoy, A., Gazyagci, S. (2011). Failure of combination therapy with imidocarb dipropionate and toltrazuril to clear Hepatozoon canis infection in dogs. Parasitology research, 109(3): 119126.

Purfield, A. E., Tidwell, R. R. and Meshnick, S. R. (2009). The diamidine DB75 targets the nucleus of Plasmodium falciparum. Malaria Journal, 8: 1-9.

Rich, M., Deighton, L. and Roberts, L. (2005). Clindamycin-resistance in methicillin-resistant Staphylococcus aureus isolated from animals. Veterinary Microbiology, 111(3-4): 237-40.

Sakuma, M., Setoguchi, A. and Endo, Y. (2009). Possible emergence of drug-resistant variants of Babesia gibsoni in clinical cases treated with atovaquone and azithromycin. Journal Veterinary Internal Medicine, 23: 493-498.

Sereno, D., Michon, P., Brajon, N. and Lemesre, J. L. (1997). Phenotypic characterization of Leishmania mexicana pentamidine-resistant promastigotes. Modulation of the resistance during in vitro developmental life cycle. $C . R$. Acad. Sci. Paris, 320: 981-987.

Soto, I. C., Fontanesi, F., Valledor, M., Horn, D., Singh, R. and Barrientos, A. (2009). Synthesis of cytochrome $\mathrm{c}$ oxidase subunit 1 is translationally downregulated in the absence of functional $\mathrm{F}_{1} \mathrm{~F}_{0}$-ATP synthase. Biochimica Biophysica Acta, 1793: 1776-1786.

Suzuki, K., Wakabayashi, H., Takahashi, M., Fukushima, K., Yabuki, A. and Endo, Y. (2007). A possible treatment strategy and clinical factors to estimate the treatment response in Babesia gibsoni infection. Journal of Veterinary Medical Science, 69: 563-568.

Teka, I. A., Kazibwe, A. J. N., El-Sabbagh, N., AlSalabi, M. I., Ward, C. P., Eze, A. A., Munday, J. C., Maser, P., Matovu, E., Barrett, M. P. and De Koning, H. P. (2011). The diamidine diminazene aceturate is a substrate for the high-affinity pentamidine transporter: implications for the development of high resistance levels in trypanosomes. Molecular Pharmacology, 80:110-116.

Townson, H., Nathan, M. B. and Zaim, M. (2005). Exploiting the potential of vector control for disease prevention. Bulletin of the World Health Organization, 83: 942-947.

Uilenberg, G. Verdiesen, P. A. H. M. and Zwart, D. (1981). Imidocarb: A chemoprophylactic experiment with Babesia canis. The veterinary quarterly, 3(3): 118-123.

Vercammen, F., Deken, R. D. and Maes, L. (1996). Prophylactic treatment of experimental canine babesiosis (Babesia canis) with doxycycline. Veterinary Parasitology, 66: 251-255.

Vial, H. J. and Gorenflot, A. (2006). Chemotherapy against babesiosis. Veterinary Parasitology, 138: 147-160.

Wiehart, U. I., Rautenbach, M. and Hoppe, H. C. (2006). Selective lysis of erythrocytes infected with the trophozoite stage of Plasmodium falciparum by polyene macrolide antibiotics. Biochemical Pharmacology, 71(6): 779-790.

Wulansari, R., Wijaya, A., Ano, H., Horii, Y., Nasu, T., Yamane, S. and Makimura, S. (2003). Clindamycin in the treatment of Babesia gibsoni infections in dogs. Journal of American Animal Hospital Association, 39: 558-562.

Wulansari, R., Wijaya, A., Ano, H., Horii, Y., Nasu, T., Yamane, S. and Makimura, S. (2003). Lymphocyte subset and specific IgG antibody levels in clindamycin treated and untreated dogs experimentally infected with Babesia gibsoni. Journal of Veterinary Medical Science, 65(5): 579-584.

Yamasaki, M., Tajima, M., Lee, K. W., Jeong, J. R., Yamato, O. and Maede, Y. (2002). Molecular cloning and phylogenetic analysis 
of Babesia gibsoni heat shock protein 70 . Veterinary Parasitology, 110: 123-129.

Yamasaki, M., Hossain, M. A., Jeong, J. R., Chang, H. S., Satoh, H., Yamato, O. and Maede, Y. (2003). Babesia gibsoni-specific isoenzymes related to energy metabolism of the parasite in infected erythrocytes. Journal of Parasitology, 89: 1142-1146.

Yamasaki, M., Takada, A., Yamato, O. and Maede, Y. (2005). Inhibition of Na, K-ATPase activity reduces Babesia gibsoni infection of canine erythrocytes with inherited high $\mathrm{K}$, low $\mathrm{Na}$ concentrations. Journal of Parasitology, 91: 1287-1292.

Yamasaki, M., Inokuma, H., Sugimoto, C., Shaw, S. E., Aktas, M., Yabsley, M. J., Yamato, O. and Maede, Y. (2007). Comparison and phylogenetic analysis of the heat shock protein 70 gene of Babesia parasites from dogs. Veterinary Parasitology, 145: 217-227.

Yamasaki, M., Kobayashi, Y., Nakamura, K., Sasaki, N., Murakami, M., Bandula, W. R. K., Ohta, H., Yamato, O., Maede, Y. and Takiguchi, M. (2011a). Babesia gibsoni: detection in blood smears and formalin-fixed, paraffin-embedded tissues using deoxyribonucleic acid in situ hybridization analysis. Experimental Parasitology, 127: 119126.

Yamasaki, M., Tamura, N., Nakamura, K., Sasaki, N., Murakami, M., Bandula W. R. K., Tamura, Y., Lim, S. Y., Ohta, H. and Takiguchi, M. (2011b). Effects and Mechanisms of action of polyene Macrolide antibiotics nystatin on Babesia gibsoni in vitro. Journal Parasitology, 97: 1190-1192.

Yamasaki, M., Harada, E., Tamura, Y., Lim, S. Y., Ohsuga, T., Yokoyama, N., Morishita, K., Nakamura, K., Ohta, H. and Takiguchi, M. (2014). In vitro and in vivo safety and efficacy studies of amphotericin B on Babesia gibsoni. Veterinary Parasitology, 205: 424-433. 
